\title{
Regulated Expression of Foreign Genes In Vivo after Germline Transfer
}

\author{
Rod S. Passman * and Glenn I. Fishman* \\ *Cardiology Division-Department of Medicine and ${ }^{\ddagger}$ Department of Molecular Genetics, Albert Einstein College of Medicine, \\ Bronx, New York 10461
}

\begin{abstract}
Tight transcriptional control of foreign genes introduced into the germline of transgenic mice would be of great experimental value in studies of gene function. To develop a system in which the spatial and temporal expression of candidate genes implicated in cardiac development or function could be tightly controlled in vivo, we have generated transgenic mice expressing a tetracycline-controlled transactivator (tTA) under the control of a rat alpha myosin heavy chain promoter (MHC $\alpha$-tTA mice), as well as mice harboring a candidate target gene implicated in the control of differentiation, Id1 (tet-Id1 mice). No expression of the target transgene was detected in any tissues of hemizygous tet-Id1 mice. Genetic crosses with MHC $\alpha$-tTA mice resulted in transactivation of the Id1 transgene, but expression was restricted to heart, where tTA was expressed. Furthermore, transactivation of the target gene was tightly and reversibly controlled by systemic therapy with tetracycline, both in utero and postnatally. These studies demonstrate the feasibility of such a binary approach for tightly controlling the timing and extent of expression of transgenes in vivo. This approach should be generally useful for the ectopic expression of candidate genes in selected tissues during delineated developmental stages. (J. Clin. Invest. 1994. 94:2421-2425.) Key words: heart • development • transgenic mice • tetracycline - transcription
\end{abstract}

\section{Introduction}

Molecular genetic manipulations of the mouse genome by gene addition (1) or homologous recombination (2) provide powerful approaches for evaluating gene function in vivo. Nonetheless, neither approach is well-suited for tight regulatory control of the gene of interest. Transgenes regulated by viral or mammalian control sequences often result in tissue and developmentally restricted expression, however, the extent and precise timing of expression only rarely can be experimentally manipulated. Gene disruption is by definition an all-or-none phenomenon. Thus, hypotheses that address whether the timing of a gene's induction or repression are important factors during development are currently difficult to test.

To modulate gene expression during distinct developmental

Address correspondence to Dr. Glenn I. Fishman, Albert Einstein College of Medicine, 1300 Morris Park Avenue, Bronx, NY 10461.

Received for publication 13 June 1994 and in revised form 25 August 1994.

J. Clin. Invest.

(c) The American Society for Clinical Investigation, Inc. $0021-9738 / 94 / 12 / 2421 / 05 \$ 2.00$

Volume 94, December 1994, 2421-2425 windows in vivo, systems for the tightly regulated spatial and temporal control of transcription are required. Several approaches have been developed which allow for exogenous regulation of gene expression in cultured mammalian cells, including the use of heavy metal or hormonally responsive promoters (3, $4)$. Other systems have relied upon modifications of bacterial genetic switches such as the lactose operon $(5,6)$ or the creation of unique chimeric proteins containing nonmammalian DNA binding and transcriptional activation domains to minimize expression of nontarget genes.

To gain tighter temporal control over mammalian gene expression, Gossen and Bujard (7) modified elements from the tetracycline-resistance operon in Tn10 of Escherichia coli. In the bacterial system, expression of tetracycline resistance genes are repressed by binding of the tetracycline repressor (tetR) to operator (tet $O$ ) sequences located within the operon. Tetracycline inhibits tetR/tet $O$ binding and derepresses transcription. By fusing tetR with the $\mathrm{COOH}$-terminal transactivation domain of herpes simplex virus VP16, a hybrid tetracycline-controlled transactivator (tTA) ${ }^{1}$ was created, which induced expression of minimal promoters harboring tet $O$ sequences. Induction was essentially completely inhibited by concentrations of tetracycline as low as $1 \mu \mathrm{g} / \mathrm{ml}$ in the media, which are similar to therapeutic antibiotic levels in the serum of rodents.

Recently, we demonstrated that target genes harboring tet $O$ sequences fused to a cytomegalovirus minimal promoter could be introduced into adult rat hearts by direct intracardiac injection and that transcription of such target genes was markedly transactivated by coinjected tTA expression plasmids ( 8 ). The level of transactivation was effectively regulated over several orders of magnitude by systemic therapy with oral tetracycline. Target gene expression could be rapidly and reversibly controlled by manipulating antibiotic treatment. However, while direct intracardiac injection of DNA with highly sensitive reporter genes is a useful technique for studies of gene transcription, the absolute number of transduced cells is limited and insufficient to influence the cardiac phenotype. Therefore, to develop an approach for the study of developmentally important genes in which both the tissue specificity and temporal profile of foreign gene expression could be highly regulated in vivo, we have examined the behavior of the tetracycline-regulated gene expression system in transgenic mice. As a model system, we have manipulated the expression of an Id 1 transgene in the heart. The Id1 gene encodes a helix-loop-helix protein which serves as a negative regulator of differentiation in several lineages, most notably during skeletal myogenesis (9). Id1 expression is temporally and spatially regulated during cardiac development, suggesting an important role during heart formation as well (10).

1. Abbreviations used in this paper: tTA, tetracycline-controlled transactivator; MHC $\alpha$, alpha myosin heavy chain. 


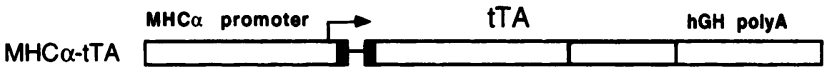

tet-dd1

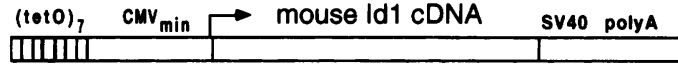

Figure 1. Plasmid constructs. MHC $\alpha$-tTA uses a rat myosin heavy chain promoter to drive tTA expression. To enhance expression, the transgene also includes a heterologous splice upstream of the tTA cDNA and human growth hormone transcriptional termination signals. The tet-Id1 target gene contains the tTA-responsive chimeric tet operon/CMV minimal promoter upstream of the mouse Id $1 \mathrm{cDNA}$. The construct includes transcriptional termination signals from the SV40 late genes.

\section{Methods}

Plasmid constructs. The tetracycline-controlled transactivator p $\alpha$-tTA has previously been described; for simplicity, in this study it is referred to as alpha myosin heavy chain (MHC $\alpha$ )-tTA (8). The tet-Idl target gene replaces the luciferase coding sequence from pUHC13-3 (7) with a SacII-EcoRI fragment from $\mathrm{pMH} 18 \Delta \mathrm{R}$, which contains the mouse Id1 cDNA (9). The MHC $\alpha$-tTA and tet-Id1 constructs are shown schematically in Fig. 1. All nucleic acid manipulations were carried out according to established protocols (11). Enzymes were obtained from New England Biolabs Inc. (Beverly, MA) unless indicated otherwise.

Tetracycline treatment. A stock solution of tetracycline hydrochloride (Sigma Chemical Co., St. Louis, MO) was prepared as $100 \mathrm{mg} /$ $\mathrm{ml}$ in $50 \%$ ethanol and diluted to its final concentration in $2.5 \%$ sucrose in water. For some studies, the parental analogue oxytetracycline (LA200; Pfizer Inc., New York) was administered ( $15 \mathrm{mg} / \mathrm{kg}$ i.p.), which was prepared by dilution of a stock solution to $2 \mathrm{mg} / \mathrm{ml}$ with water.

Transgenic mouse production. The MHC $\alpha$-tTA plasmid was linearized at the unique XmnI site in the vector backbone and then purified from solution using sodium iodide and silica beads (Gene Clean; Bio 101, La Jolla, CA). The tet-Id1 transgene was separated from vector sequences by agarose gel electrophoresis after digestion with XhoI and BstEII and similarly purified. Transgene DNA was then equilibrated with injection buffer ( $10 \mathrm{mM}$ Tris ( $\mathrm{pH} 7.4$ ), $0.1 \mathrm{mM}$ EDTA) by spin dialysis using a filter (Microcon; Amicon Corp., Danvers, MA). Pronuclear injection was carried out in the Albert Einstein College of Medicine Transgenic Mouse Facility according to standard techniques, into a B6/ $\mathrm{CBAF}_{1}$ background (12).

Hybridization analyses. Genomic DNA was prepared from tail biopsies and $10 \mu \mathrm{g}$ of DNA was digested with the appropriate restriction endonuclease and size-fractionated on $1 \%$ agarose gels. After denaturation and neutralization, DNA was transferred to nylon membranes (Hybond N; Amersham International, Buckinghamshire, UK) by capillary transfer and subsequently cross-linked by ultraviolet irradiation (Stratalinker; Stratagene Inc., La Jolla, CA).

Total cellular RNA was prepared from tissue samples by the singlestep method (13). For each sample, $\sim 10 \mu \mathrm{g}$ of total RNA was sizefractionated on $0.8 \%$ agarose/formaldehyde gels and transferred and cross-linked as described above.

To identify tTA transgene expression, a 1-kb EcoRI-BamHI cDNA fragment from pUHD 13-3 including the tTA coding region was used. To specifically detect Id 1 transgene transcripts without cross-hybridization with endogenous Id sequences, a 166-bp EcoRI-HincII cDNA fragment from the tet-Idl plasmid was used which contained sequences from the SV40 late genes included in the processed transcript. Complementary DNA probes were labeled to high specific activity by random priming using a commercial kit (Prime-It II; Stratagene Inc.). Membranes were hybridized under high stringency conditions using a $50 \%$ formamide solution at $42^{\circ} \mathrm{C}$ and were washed to a final stringency of

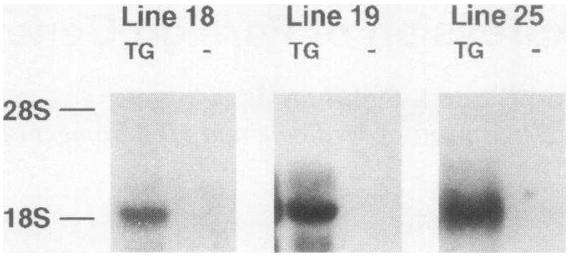

Figure 2. Cardiac expression of the MHC $\alpha$-tTA Transgene. Newborn offspring from matings between founders and nontransgenic littermates were killed and total RNA prepared from individual hearts and genomic DNA prepared from tail biopsies. Transgene status was determined by Southern blot analysis. Representative Northern blot analysis of transgenic (TG) and nontransgenic (-) progeny from lines 18, 19, and 25 are shown. Each line expresses the expected $\sim 1.5$-kb transcript, although the abundance varied among each line.

$0.2 \times \mathrm{SSC}$ and $0.2 \%$ SDS at $65^{\circ} \mathrm{C}$. Autoradiographic signals were quantified by scanning laser densitometer (Molecular Dynamics, Inc., Sunnyvale, CA).

\section{Results}

Generation of transgenic mice. Potential founders carrying the MHC $\alpha$-tTA transgene were identified by Southern blot analysis of tail DNA (not shown). 4 of 25 live births carried the transgene. Although not directly quantified, based upon the intensity of the hybridization signal, all lines appeared to have integrated multiple copies of the transgene. In contrast, a single line harboring the tet-Id 1 construct was identified from 46 births. Based upon a comparison with the endogenous Id1 gene signal intensity, the tet-Id1 transgene was present in this line as a single haploid copy. For some studies, tet-Idl transgenic mice were bred to homozygosity, as determined by quantification of the Southern blot hybridization signal and their capacity to pass on the transgene to all progeny.

Cardiac expression of the $M H C \alpha-t T A$ transgene. Expression of the MHC $\alpha$-tTA transgene was initially evaluated in the hearts of postnatal day 1 transgenic mice. For each line, founders were mated with nontransgenic littermates and total cellular RNA prepared individually from the hearts of each F1 offspring. As shown in Fig. 2, the predicted 1.5-kb band was observed in lines 18, 19, and 25. Overall, expression of the transgene was similar among the three lines tested, but there were rare individuals within litters with markedly reduced expression, suggesting either background strain effects or possibly the influence of bacterial vector sequences included in this transgene. To date, the line 16 founder has not successfully transmitted the transgene and presumably did not attain germline transmission.

tTA-dependent expression of the tet-Idl target gene. Expression of the Idl target was first evaluated in hemizygous mice carrying only the tet-Id 1 transgene. No expression of the transgene was observed by Northern blot analysis in any of the tissues sampled, including brain, heart, liver, kidney, hindlimb muscle, spleen, or tongue (data not shown).

To determine the effects of introduction of the MHC $\alpha$-tTA transgene into the tet-Id1 background, crosses between the MHC $\alpha$-tTA line 25 and tet-Id1 lines were carried out. F1 progeny were killed on postnatal day 1 and genotyped for each of the two transgenes, as shown in Fig. 3A. Total cardiac RNA was prepared from each of the offspring and evaluated for expression of both tTA and the Idl target gene $(B)$. Only those 
A.

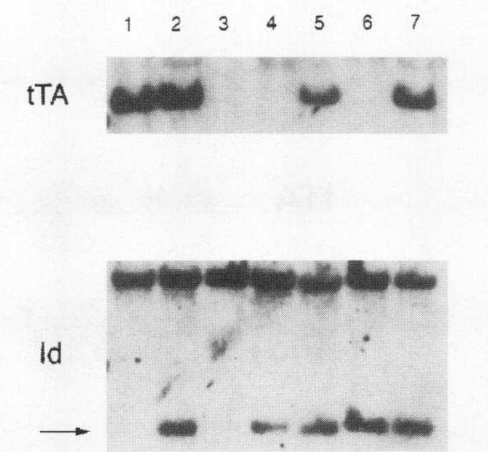

B.

.

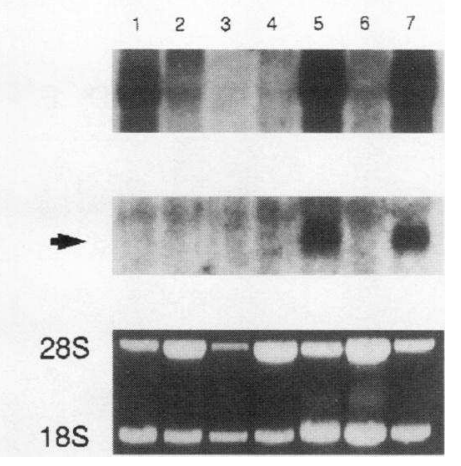

Figure 3. Transactivator-dependent expression of the tetIdl target gene $(A)$ Southern blot analysis. Matings were prepared between the MHC $\alpha$-tTA line 25 founder and a hemizygous tet-Id 1 target gene mouse. Offspring were killed at the time of birth. Genomic DNA was prepared from tail biopsies of each animal and evaluated for the presence of each of the two transgenes, tTA and Id 1 (arrow). F1 offspring 2, 5, and 7 are doubly transgenic. $(B)$ Northern blot analysis. Total RNA was prepared from the hearts of each of the offspring and evaluated for expression of the MHC $\alpha$-tTA transactivator and tet-Id 1 target gene. F1 offspring 1, 5, and 7 express the transactivator at high levels, whereas animal 2 shows minimal expression. Although five animals $(2,4,5,6$, and 7$)$ harbor the tet-Id1 target transgene, only those which also express the transactivator ( 5 and 7 ), accumulate the target transcript (arrowhead). mice which were compound hemizygous for both transgenes and expressed tTA accumulated the target gene transcript. Although animal 2 was doubly transgenic, it failed to express significant tTA mRNA and subsequently did not transactivate the tet-Id 1 target gene. Seven additional doubly transgenic mice resulting from crosses between the MHC $\alpha$-tTA line 25 founder and tet-Id 1 females were identified. These compound heterozygote offspring were analyzed at ages ranging from postnatal day $1-35$. In all cases, concordant expression of the transactivator and target genes was observed (not shown).

Cardiac-specific expression of the target gene. The cardiacspecificity of tTA expression under the control of the MHC $\alpha$ promoter was reflected in the pattern of tet-Idl target gene transactivation, as shown in Fig. $4(A)$. In doubly transgenic mice, expression of both transactivator and target genes was restricted to heart; no expression of the tet-Id1 target gene was observed in brain, kidney, liver, hindlimb muscle, spleen, or tongue.

Inhibition of transactivation in compound transgenic mice. We next determined whether tetracycline therapy could reversibly control the expression of the target gene and regulate tTAdependent transactivation in the hearts of compound transgenic mice. To increase the proportion of doubly transgenic offspring, the MHC $\alpha$-tTA line 25 founder was mated with homozygous tet-Id1 mice. To control target gene expression during cardiac development, maternally administered tetracycline must effectively cross into the fetal circulation and enter the developing heart. Therefore, we first tested the effects of maternally administered tetracycline on target gene expression in her progeny. In contrast to the results obtained in the absence of tetracycline therapy (see Fig. 3), no target gene transactivation was observed in the hearts of doubly transgenic offspring for the first $72 \mathrm{~h}$ after birth, despite abundant expression of tTA (Fig. $4 \mathrm{~B}$, lanes $A-C$ ). Similar results were observed in eight additional compound heterozygous mice analyzed within $72 \mathrm{~h}$ after birth (not shown). To determine whether repression was reversible, additional doubly transgenic offspring were obtained from the same mating pair, which had again received tetracycline throughout gestation. Three doubly transgenic offspring were identified. The mother and these progeny remained off additional tetracycline therapy from the time of birth until postnatal day 10. Analysis at this time point demonstrated abundant target gene expression (lane $D$ ). Moreover, additional antibiotic therapy from postnatal days 10-13 again blocked target gene ex- pression, to levels undetectable by Northern blot analysis (lane $E$ ). The third doubly transgenic mouse expressed negligible tTA mRNA and as expected, did not accumulate the target gene (not shown). Thus, cardiac-specific target gene expression was highly and reversibly responsive to tetracycline treatment in vivo, both prenatally, in the developing heart, as well as postnatally.

Finally, to determine whether repression of target gene transactivation in utero by maternal administration of tetracycline could be reversed more rapidly, the mating pair was supplemented with only $0.1 \mathrm{mg} / \mathrm{ml}$ tetracycline in the drinking water during gestation, which was stopped at the time of birth. Compound heterozygous offspring were identified and analyzed 24,48 , or $120 \mathrm{~h}$ postnatally, as shown in Fig. $4 C$. In contrast to the results obtained with the higher dosage of tetracycline, very weak expression of the tet-Idl target gene was observed as early as $24 \mathrm{~h}$ after discontinuation of antibiotic treatment (lanes $A$ and $B$ ) and increased approximately 20 -fold by day 5 (lane $D$ ).

\section{Discussion}

In these studies, we have demonstrated the functionality of the tetracycline-regulated gene expression system directly in transgenic mice. First, we have shown that transgenic mice carrying only the tTA-dependent target gene are transcriptionally silent, at least by the sensitivity of Northern blot analysis. Second, we have shown that introduction of the chimeric tTA protein by genetic means effectively transactivates the normally silent target gene. Finally, we have demonstrated that tetracycline treatment effectively represses tTA-dependent transactivation and that this effect is reversible.

The magnitude of target gene expression in the absence of expressed transactivator is a critical parameter. For some applications, even relatively minor levels of tTA-independent target gene transcription will be unacceptable. Examples of such studies might include targeted expression of toxins in fully differentiated adult cells or complex genetic complementation experiments. In our studies, we observed no detectable target gene expression, at least by the limited sensitivity of hybridization analysis. Conceivably, independent lines of mice will have varying degrees of baseline transcription, reflecting the site of chromosomal integration. Hence, investigators may need to screen several target gene founders by highly sensitive techniques, 


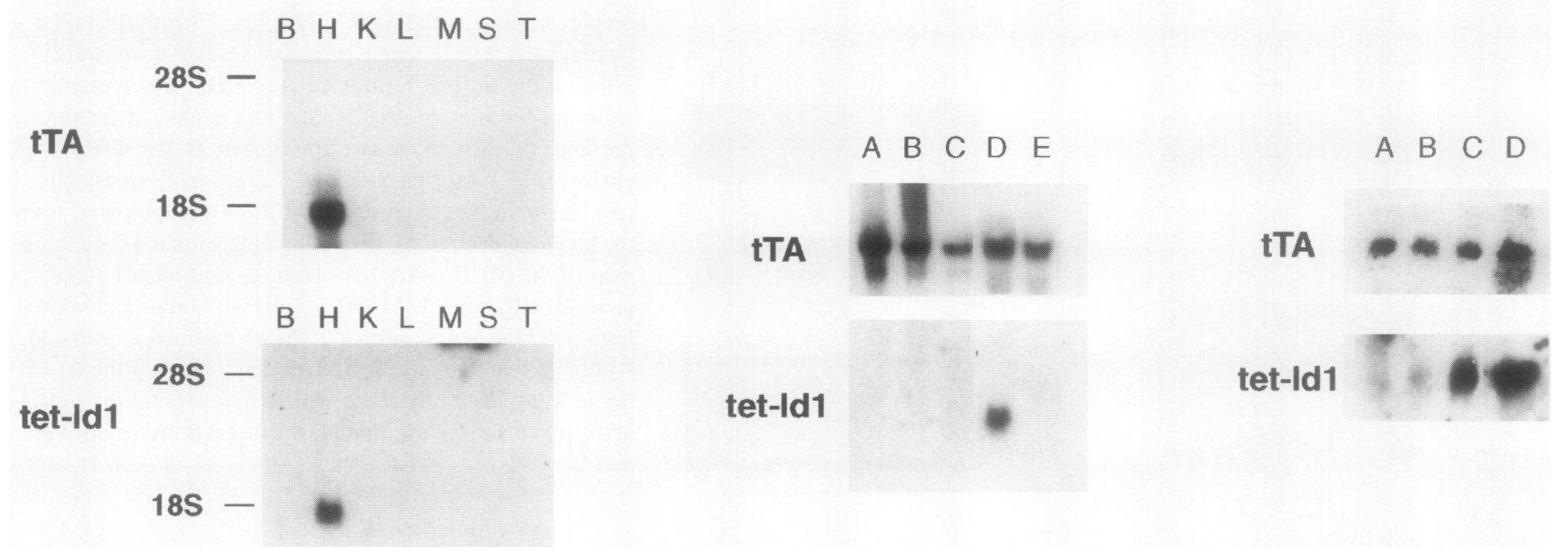

Figure 4. Cardiac-specific target gene transactivation and repression. (A) Tissue-specific transactivation. Offspring from a cross between the MHC $\alpha$ tTA line 25 founder and a homozygous tet-Id1 female were genotyped. A doubly transgenic mouse was identified and total RNA prepared from selected tissues and analyzed for expression of the transactivator (tTA) and tet-Id1 target gene (tet-Id1) by Northern blot analysis. Expression of both genes is restricted to the heart (H); no expression is observed in brain (B), kidney (K), liver (L), hindlimb muscle (M), spleen (S), or tongue (T). (B) Tetracycline-dependent repression. Matings were set up between the MHC $\alpha$-tTA line 25 founder and homozygous tet-Id1 females. The mating pairs were supplemented with oral tetracycline therapy $(1 \mathrm{mg} / \mathrm{ml}$ in the drinking water) both before and throughout the pregnancies. At the time of birth, antibiotic therapy was discontinued. Transactivator (tTA) and target gene (tet-Id1) expression were analyed in doubly trangenic progeny at $1,2,3$, or $10 \mathrm{~d}$ after birth $(A-D)$. A fifth mouse received three consecutive daily intraperitoneal doses of oxytetracycline beginning on postnatal day 10 and was then analyzed on postnatal day $13(E)$. Target gene expression is fully and reversibly repressed during gestation and immediately postpartum by tetracycline treatment. $(C)$ Induction of target gene expression. Identical matings were set up as above and the mating pairs were supplemented with low dose oral tetracycline therapy $(0.1 \mathrm{mg} / \mathrm{ml}$ in the drinking water) both before and throughout the pregnancies. Antibiotic therapy was discontinued at the time of birth. Transactivator (tTA) and target gene (tet-Id1) expression were analyzed in doubly transgenic progeny at $24(A$ and $B), 48(C)$ or $120(D)$. Target gene expression rises about 20-fold during this time period.

such as reverse-transcription PCR, to identify lines which are truly transcriptionally inactive in the absence of tTA.

Several genetic systems already exist in which transgene expression can be modified in vivo. Binary approaches, such as the GAL4 system recently described, allow for target genes to be specifically transactivated in doubly transgenic progeny (14). However, in this approach, the extent of transactivation is unregulated and target genes cannot be specifically repressed or activated during critical developmental stages. Alternative approaches, such as the cre-lox system of bacteriophage P1 allow for the precise recombination of foreign genetic elements in vivo, resulting in either gene activation or disruption (15). However, if cre is genetically expressed under the control of a cellular promoter, the onset of recombinase activity is limited to a single developmental stage and alternatively, if the recombinase is introduced by a viral vector, the recombination event may not occur uniformly or be restricted to the tissue of interest.

In our experiments, we directed tTA expression to the heart by means of a cardiac-specific myosin heavy chain promoter. Therefore, not only could expression of the target gene be temporally and quantitatively controlled by tetracycline administration, but its spatial behavior was delineated as well. This approach enabled us to specifically transactivate the Id 1 transgene only within the cardiac myocyte. It should be noted that expression of tTA under the control of the short MHC $\alpha$ promoter showed some variability within individual litters. This may reflect background effects, or more likely, the inclusion of bacterial sequences within the injected transgene. To minimize this variability, we have now constructed transgenes in which additional 5' flanking sequences from the MHC $\alpha$ gene are included and all bacterial sequences have been removed. Others have reported that these modifications result in consistently high levels of cardiac-specific transgene expression in vivo (16).

Normally, Id1 expression is observed in all embryonic cell layers by $7.5 \mathrm{~d}$ p.c., but disappears in the heart as gastrulation ends. By 10.5 d p.c., however, Id expression is strong in the endocardial cushion region and by $13.5 \mathrm{~d}$ p.c., the transcript is also observed in the valves and outflow tract. By the end of gestation, Id expression within the heart is restricted to the cardiac valves (10). Thus, the endogenous Id1 gene shows a complex temporal and spatial pattern of expression suggesting an important role in cardiac development. In this study, we have not yet addressed potential phenotypic effects of Id 1 target gene transactivation. Given the concordance between transactivator and target gene expression in postnatal compound heterozygotes, it is likely that the tet-Id1 target gene is expressed during embryogenesis, since the MHC $\alpha$ promoter we have used to control tTA expression is quite active during prenatal heart formation (not shown). Thus, aberrant Idl expression during the latter part of cardiac development is presumably not embryonic lethal. Conceivably, posttranscriptional mechanisms regulate the accumulation of Id1 protein, mitigating the effects of target gene transactivation. Clearly, additional studies will be required to determine whether more subtle phenotypic alterations are present in these transgenic mice.

In summary, we have demonstrated the functionality of the tetracycline-regulated gene expression system in vivo after germline transfer. The ability to spatially and temporally control foreign gene expression following germline transfer should prove exceedingly useful for in vivo developmental studies. 
The consequences of ectopic expression of candidate genes in selected tissues during delineated developmental stages can now be experimentally tested. In addition, one can now envision selective rescue of stage-specific developmental defects resulting from targeted gene disruptions.

\section{Acknowledgments}

We thank H. Bujard and M. Gossen for plasmids pUHD10-1, pUHC133 and pUHD15-1; R. Benezra for the Idl cDNA; V. Mahdavi for the MHC $\alpha$ genomic clone; C. Branigan and Y. Zhihui for expert technical assistance; and J. Horner for his help in the transgenic mouse facility.

This was supported in part by a National Institutes of Health Physican Scientist Award HL-02391 and a Grant-in-Aid from the American Heart Association to G. I. Fishman and National Institutes of Health postdoctoral training grant 5T32HL076 to R. S. Passman.

\section{References}

1. Brinster, R. L., M. Y. Chen, M. E. Trumbauer, A. W. Sevear, R. Warren, and R. D. Palmiter. 1981. Somatic expression of MSU-TU in mice following injection of a fusion gene into eggs. Cell. 27:223-231.

2. Thomas, K. R., and M. R. Capecchi. 1987. Site-directed mutagenesis by gene targeting in mouse embryo-derived stem cells. Cell. 51:503-512.

3. Hamer, D. H., and M. Walling. 1982. Regulation in vivo of a mammalian gene: cadmium induction of a mouse metallothionein gene in SV40 vectors. $J$. Mol. Appl. Genet. 1:273-288.

4. Payver, F., D. DeFranco, G. L. Firestone, B. Edgar, O. Wrange, S. Okret, J. A. Gustafsson, K. R. Yamamoto. 1983. Sequence-specific binding of glucocorti- coid receptor to MTV DNA at sites within and upstream of the transcribed region. Cell. 35:369-377.

5. Labow, M. A., S. B. Baim, T. Shenk, A. J. Levine. 1990. Conversion of the lac repressor into an allosterically regulated transcriptional activator for mammalian cells. Mol. Cell. Biol. 10:3343-3356.

6. Baim, S. B., M. A. Labow, A. J. Levine, and T. Shenk. 1991. A chimeric mammalian transactivator based on the lac repressor that is regulated by temperature and isopropyl-b- D-thiogalactopyranoside. Proc. Natl. Acad. Sci. USA. 88:5072-7076.

7. Gossen, M., and H. Bujard. 1992. Tight control of gene expression in mammalian cells by tetracycline-responsive promoters. Proc. Natl. Acad. Sci. USA. 89:5547-51.

8. Fishman, G. I., M. L. Kaplan, and P. M. Buttrick. 1994. Tetracyclineregulated cardiac gene expression in vivo. J. Clin. Invest. 93:1864-1868.

9. Benezra, R., R. L. Davis, D. Lockshon, D. L. Turner, and H. Weintraub. 1990. The protein Id: a negative regulator of helix-loop-helix DNA binding proteins. Cell. 61:49-59.

10. Wang, Y., R. Benezra, and D. A. Sassoon. 1992. Id expression during mouse development: a role in morphogenesis. Dev. Dyn. 194:222-230.

11. Ausebel, F. M., R. Brent, R. E. Kingston, D. D. Moore, J. G. Seidman, J. A. Smith, and K. Struhl, editors. 1987. Current Protocols in Molecular Biology. Wiley Interscience, New York.

12. Hogan, B., F. Costantini, and E. Lacy. 1986. Manipulating the Mouse Embryo: A Laboratory Manual. Cold Spring Harbor Laboratory, Cold Spring Harbor, NY.

13. Chomczynsky, P., and N. Sacchi. 1987. Single-step method of RNA isolation by acid guanidinium thiocyanate phenol-chloroform extraction. Anal. Biochem. 162:156-159.

14. Ornitz, D. M., R. W. Moreadith, and P. Leder. 1991. Binary system for regulating transgene expression in mice: targeting int-2 gene expression with yeast GAL4/UAS control elements. Proc. Natl. Acad. Sci. USA. 88:698-702.

15. Orban, P. C., D. Chui, and J. D. Marth. 1992. Tissue- and site-specific DNA recombination in transgenic mice. Proc. Natl. Acad. Sci. USA. 89:68616865 .

16. Subramaniam, A., W. K. Jones, J. Gulick, S. Wert, J. Neumann, and J. Robbins. 1992. Tissue-specific regulation of the alpha-myosin heavy chain gene promoter in transgenic mice. J. Biol. Chem. 266:24613-20. 